\title{
An enhanced recovery after surgery pathway: association with rapid discharge and minimal complications after anterior cervical spine surgery
}

\author{
Ellen M. Soffin, MD, PhD, ${ }^{1,2}$ Douglas S. Wetmore, MD, ${ }^{1,2}$ Lauren A. Barber, MD, ${ }^{3}$ \\ Avani S. Vaishnav, MBBS, ${ }^{3}$ James D. Beckman, MD, ${ }^{1,2}$ Todd J. Albert, MD, ${ }^{3,4}$ \\ Catherine H. Gang, MPH, ${ }^{3}$ and Sheeraz A. Qureshi, MD ${ }^{3,4}$ \\ 'Department of Anesthesiology, Critical Care \& Pain Management, Hospital for Special Surgery; ${ }^{2}$ Department of Anesthesiology, \\ Weill Cornell Medicine; ${ }^{3}$ Department of Orthopaedic Surgery, Hospital for Special Surgery; and ${ }^{4}$ Department of Orthopaedic \\ Surgery, Weill Cornell Medicine, New York, New York
}

OBJECTIVE Enhanced recovery after surgery (ERAS) pathways are associated with improved outcomes, lower morbidity and complications, and higher patient satisfaction in multiple surgical subspecialties. Despite these gains, there are few data to guide the application of ERAS concepts to spine surgery. The authors report the development and implementation of the first ERAS pathway for patients undergoing anterior cervical discectomy and fusion (ACDF) and cervical disc arthroplasty (CDA).

METHODS This was a retrospective cohort study of prospectively collected data. The authors created a multidisciplinary pathway based on best available evidence for interventions that positively influence outcomes after anterior cervical spine surgery. Patients were followed prospectively up to postoperative day 90 . Patient data were collected via electronic medical record review and included demographics, comorbidities, baseline and perioperative opioid use, postoperative complications, and length of hospital stay (LOS). ERAS process measures and compliance with pathway elements were also tracked.

RESULTS Thirty-three patients were cared for under the pathway ( $n=25$ ACDF; $n=8$ CDA). The median LOS was 416 minutes (interquartile range [IQR] 210-1643 minutes). Eight patients required an extended stay-longer than 23 hours. Reasons for extended admission included pain $(n=4)$, dyspnea $(n=1)$, hypoxia $(n=1)$, hypertension $(n=1)$, and dysphagia $(n=1)$. The median LOS for the 8 patients who required extended monitoring prior to discharge was 1585 minutes (IQR 1423-1713 minutes). Overall pathway compliance with included process measures was $85.6 \%$. The median number of ERAS process elements delivered to each patient was 18. There was no strong association between LOS and number of ERAS process elements provided (Pearson's $r=-0.20$ ). Twelve percent of the cohort was opioid tolerant on the day of surgery. There were no significant differences between total intraoperatively or postanesthesia care unitadministered opioid, or LOS, between opioid-tolerant and opioid-naïve patients. There were no complications requiring readmission.

CONCLUSIONS An ERAS pathway for anterior cervical spine surgery facilitates safe, prompt discharge. The ERAS pathway was associated with minimal complications, and no readmissions within 90 days of surgery. Pain and respiratory compromise were both linked with extended LOS in this cohort. Further prospective studies are needed to confirm the potential benefits of ERAS for anterior cervical spine surgery, including longer-term complications, cost, and functional outcomes.

https://thejns.org/doi/abs/10.3171/2019.1.FOCUS18643

KEYWORDS enhanced recovery after surgery; ERAS; anterior cervical discectomy and fusion; cervical disc replacement; perioperative factors

ABBREVIATIONS ACDF = anterior cervical discectomy and fusion; CDA = cervical disc arthroplasty; ERAS = enhanced recovery after surgery; IQR = interquartile range; LOS = length of hospital stay; MIS = minimally invasive surgery; MMA = multimodal analgesia (defined as use of $>1$ nonopioid analgesic agent); NRS = numeric rating scale; $\mathrm{PACU}=$ postanesthesia care unit; $\mathrm{PONV}=$ postoperative nausea and vomiting.

SUBMITTED November 27, 2018. ACCEPTED January 23, 2019.

INCLUDE WHEN CITING DOI: 10.3171/2019.1.FOCUS18643. 
$\mathrm{E}$ NHANCED recovery after surgery (ERAS) is a comprehensive perioperative care model that optimizes outcomes via standardized, evidence-based interventions..$^{13}$ A major goal of ERAS is to maintain organ homeostasis and minimize the surgical stress response, so that patients experience a more rapid return to baseline function after surgery. Within orthopedic surgery, ERAS protocols have been widely applied to elective total joint arthroplasty. $9,29,30,32$ Consistent with reports from other surgical procedures, orthopedic ERAS protocols are associated with improved outcomes, shorter length of hospital stay (LOS), fewer complications, and significant cost savings. ${ }^{19}$

Despite these positive results, data on the safety, feasibility, and outcomes achieved with ERAS for spine surgery are limited. Although proposals for the suitability, ${ }^{34}$ pathway composition, ${ }^{16}$ and development ${ }^{1}$ of ERAS for complex spine surgery have been made, associated experience and outcomes are currently lacking. One description of an ERAS pathway for patients undergoing surgery for metastatic spine tumor concluded that there was a trend toward better pain scores and decreased opioid consumption in the ERAS cohort, but no significant differences in LOS, 30-day readmission, or complication rates between ERAS and standard care. ${ }^{8}$ Two retrospective series described ERAS for spine surgery performed using minimally invasive surgery (MIS) techniques: one as applied to lumbar fusion, ${ }^{35}$ and the other to lumbar decompression. ${ }^{31}$ Both reports associated ERAS with short LOS and minimal complications, but each pathway included different care elements, different numbers of elements, and divergent rationales for the selection of included elements.

The goal of the present study was to adapt the ERAS model for lumbar decompression surgery to patients undergoing anterior cervical spine surgery. Here, we describe how the pathway was tailored and applied to 33 patients presenting for anterior cervical discectomy and fusion (ACDF) or cervical disc arthroplasty (CDA) in the first 6 months after implementation of the ERAS pathway. We additionally report our initial experience and patient outcomes, including high rates of ERAS compliance, short duration of hospital stay, and minimal complications.

\section{Methods}

This study was conducted under IRB approval.

\section{Study Design and Data Collection}

We performed a retrospective cohort study of prospectively collected data. The ERAS pathway for anterior spine surgery was implemented in August 2017. Cases treated after implementation were prospectively logged into a database. We analyzed all consecutive cases performed over the first 6 months after pathway implementation (August 2017 to January 2018). The pathway was applied to all patients presenting for ACDF or CDA without applying any patient-related inclusion or exclusion criteria. The demographic information, diagnoses, operative and anesthetic details, and ERAS elements provided were collected by manual review of the electronic medical record. The primary outcome was LOS, defined as readiness for discharge from the postanesthesia care unit (PACU). Patients were considered ready for discharge from the PACU when they achieved a modified Aldrete score $\geq 9$ after a minimum of 5 hours of observation for airway compromise. ${ }^{21}$ Secondary outcomes included the following: 1) pathway compliance (defined as the number of ERAS process elements delivered to each patient); 2) the incidence of and reasons for LOS exceeding 23 hours; and 3) the prevalence of opioid tolerance at baseline, and the effect of opioid tolerance on outcomes.

\section{Statistical Methods}

Descriptive statistics were used for the study population. Demographic and clinical characteristics were summarized using counts and percentages for categorical variables. Continuous variables were expressed as the mean $\pm \mathrm{SD}$ for normally distributed data or the median and interquartile range (IQR) for skewed data. Unpaired t-tests were calculated to assess differences between continuous variables, and the chi-square test was used for categorical variables. A Pearson product-moment correlation coefficient was calculated to assess the relationship between the number of ERAS components provided and LOS.

\section{Pathway Design}

We solicited multidisciplinary input for an agreement on the components of care for the ERAS pathway. Expertise was represented from anesthesiology, surgery, physical therapy, pharmacy, nutrition, and nursing. We previously described the development and implementation of an evidence-based ERAS pathway for lumbar decompression. ${ }^{31}$ Patient demographics, comorbidities, and procedural details are presented in Table 1. Using this pathway as a template, we adjusted components of care to address the distinct perioperative needs of patients undergoing ACDF or CDA via an MIS approach (Table 2).

In brief, preoperatively, all patients were provided education regarding expected LOS ( $<24$ hours); anticipated postoperative pain; the role of multimodal analgesia (MMA, defined as use of $>1$ nonopioid analgesic agent) and opioids in pain management; how to grade pain; how reported pain scores translate into analgesic choices; goals for early physical therapy and mobilization; and instructions for surgical follow-up. Procedure-specific education for the ERAS for cervical spine surgery included the possibility of prolonged intubation and a discussion regarding risks of dysphagia. Oral intake guidelines were provided, which allowed solid food until 6 hours prior to surgery and clear liquids up to 4 hours prior to surgery. All patients were provided with a clear carbohydrate beverage (Ensure Clear, $10 \mathrm{fl} \mathrm{oz}$ ) to consume 4 hours prior to surgery.

On the day of surgery preemptive analgesia was given in the holding area (acetaminophen, $1000 \mathrm{mg}$ oral; gabapentin, $300 \mathrm{mg}$ oral) unless contraindicated or refused by the patient. All patients were assessed for risk of postoperative nausea and vomiting (PONV). The number and class of agent to be provided was determined according to established guidelines and risk factors for PONV. ${ }^{7} \mathrm{~Pa}-$ tients deemed at high risk for PONV were prescribed a prophylactic scopolamine patch (1.5 $\mathrm{mg}$ transdermal) to 
be applied in the holding area, provided there was no contraindication. ${ }^{3}$

Intraoperative care elements emphasized opioid-sparing anesthetic and analgesic regimens, prophylaxis against PONV, and timely antibiotic administration. Given that ACDF and CDA are not associated with significant fluid and volume shifts or high anticipated blood loss, intravenous fluid administration was not performed via strict goal-directed parameters. Additionally, a mildly fluid-restricted regimen is associated with protection against postACDF airway compromise. ${ }^{11}$ Accordingly, intravenous fluid administration was titrated to a total intraoperative goal of approximately $10-15 \mathrm{ml} \cdot \mathrm{kg}^{-1}$. Maintenance of hemodynamic goals (mean arterial pressure within $20 \%$ of baseline) was achieved with ephedrine administration (5$10 \mathrm{mg}$, intravenous bolus doses) as needed. Patients were warmed with convective plus intravenous fluid warmers with a goal core temperature between $36.0^{\circ} \mathrm{C}$ and $37.0^{\circ} \mathrm{C}$.

A standardized surgical approach was used. Left-sided horizontal incision with the standard Smith-Robinson approach was used for access to the anterior cervical spine in all cases. Self-retaining retractors with narrow, blunt blades that only retracted along the length of the operative disc space were used. Distraction pins were used in all cases, as was an operating microscope. After completion of decompression, either a disc replacement implant or fusion device was placed in the disc space. All interbody devices used were zero-profile types of implants. At the conclusion of the procedure, but before skin closure, $1 \mathrm{ml}$ of $40 \mathrm{mg} / \mathrm{ml}$ methylprednisolone was directly delivered onto the outer wall of the esophagus where the retractors had been used. No surgical drains or Foley catheters were placed. In contrast to our ERAS pathway for lumbar surgery, procedure-specific evidence supported the inclusion of topical methylprednisolone for anterior cervical procedures. ${ }^{26,37}$

Postoperatively, patients were encouraged to attempt oral intake as soon as they had recovered from anesthesia and a bedside swallow evaluation was considered satisfactory by the responsible nurse. A 5-hour mandatory PACU stay for observation and 2 swallowing tests (i.e., patient has to eat twice) are required for all patients before discharge. Physical therapy was initiated and emphasized an early ambulation goal (within 2 hours of PACU arrival). Multimodal opioid-sparing analgesia was provided, according to an electronic order set developed for ERAS patients. Numeric rating scale (NRS) scores of pain were recorded at regular intervals during the PACU stay. The first score was taken after recovery from anesthesia, and then at 30-minute intervals until the NRS score was $\leq 4$ on 2 successive occasions. Thereafter, NRS scores were recorded hourly for the duration of the PACU admission, or more frequently (every 30 minutes) if the patient reported worsening pain (i.e., $\mathrm{NRS} \geq 5$ ).

Analgesics were administered according to pain state, where NRS score $<5$ was treated with acetaminophen, NSAIDs, or nonpharmacological intervention (positioning change, reeducation, and reassurance). NSAIDs were included in this ERAS pathway, after a consideration that the opioid-sparing and analgesic benefits of NSAIDs outweighed the risk of compromised fusion ${ }^{20,27}$ or platelet
TABLE 1. Demographics in 33 patients who underwent anterior cervical spine surgery

\begin{tabular}{|c|c|c|c|}
\hline Variable & ACDF & CDA & $p$ Value \\
\hline No. of patients & 25 & 8 & \\
\hline Age in yrs, median (IQR) & $58(47,61)$ & $44(33,50)$ & $<0.05$ \\
\hline Male sex, no. (\%) & $13(52)$ & $2(25)$ & $<0.05^{*}$ \\
\hline BMI $\left(\mathrm{kg} / \mathrm{m}^{2}\right)$, mean $\pm \mathrm{SD}$ & $27.2 \pm 4.4$ & $25.2 \pm 4.1$ & NS \\
\hline ASA grade, no. (\%) & & & $N S^{*}$ \\
\hline 1 & $15(60)$ & $6(75)$ & \\
\hline II & $10(40)$ & $2(25)$ & \\
\hline III & 0 & 0 & \\
\hline Diabetes mellitus & 1 & 1 & \\
\hline Hypertension & 2 & 0 & \\
\hline Current smoker & 3 & 0 & \\
\hline COPD & 1 & 0 & \\
\hline \multicolumn{4}{|l|}{ No. of levels (\%) } \\
\hline 1 & $12(48)$ & $8(100)$ & $<0.001^{*}$ \\
\hline 2 & $12(48)$ & 0 & \\
\hline 3 & $1(4)$ & 0 & \\
\hline $\begin{array}{l}\text { Duration of surgery } \\
\text { (mins), mean } \pm \mathrm{SD}\end{array}$ & $73.0 \pm 21.9$ & $60.0 \pm 6.9$ & NS \\
\hline $\begin{array}{l}\text { IOP blood loss in ml, } \\
\text { median (IQR) }\end{array}$ & $25(25,50)$ & $20(20,25)$ & NS \\
\hline $\begin{array}{l}\text { IOP intravenous fluid in } \\
\mathrm{ml} \text {, median (IQR) }\end{array}$ & $900(500,1000)$ & $750(500,1000)$ & NS \\
\hline
\end{tabular}

$\mathrm{ASA}=$ American Society of Anesthesiologists; $\mathrm{COPD}=$ chronic obstructive pulmonary disease; $\mathrm{IOP}=$ intraoperative; NS = not significant.

* Chi-square test; all other comparisons via unpaired t-test. Comorbid conditions were not formally analyzed due to low incidence.

dysfunction and bleeding. ${ }^{33}$ An NRS score $\geq 5$ was treated with tramadol (50 $\mathrm{mg} \times 2$ doses as needed). Patients who required $>2$ doses of tramadol and/or reported an NRS score $\geq 8$ were provided with oxycodone (5 mg every 3 hours as needed). After 5 hours of observation in PACU, patients were evaluated by the anesthesiologist for readiness for discharge, according to Aldrete criteria, ${ }^{21}$ including tolerating a regular diet, ambulating without assistance, adequate pain control on oral analgesics, and with a clear understanding of the follow-up plan for care and contact details in the event of concerns, questions, or complications.

\section{Results}

\section{Demographic Data}

We included 33 consecutive cases performed between August 2017 and January 2018 in the analysis. Patient demographics, comorbidities, and procedural details are presented in Table 1. Twenty-five patients underwent ACDF and 8 patients underwent CDA. All patients presenting for CDA underwent single-level surgery. Twelve patients underwent 1-level ACDF, 12 patients had 2-level ACDF, and 1 patient had 3-level ACDF. Patients presenting for ACDF were older (median 58 years [IQR 47-61 years]) than those presenting for CDA (median 44 years [IQR 33-50 
TABLE 2. The Hospital for Special Surgery ERAS for anterior cervical spine pathway components

\begin{tabular}{|c|c|}
\hline Op Stage & Pathway Component* \\
\hline \multirow[t]{7}{*}{ Preop } & Patient education and expectation setting \\
\hline & Anticipated recovery, pain trajectory, and LOS \\
\hline & Physical therapy and rehabilitation goals \\
\hline & Role of MMA and opioids in periop analgesia \\
\hline & Emphasis on the role of the patient in their own recovery \\
\hline & Nutrition \\
\hline & $\begin{array}{l}\text { NPO guidelines provided (verbal and written): solids until } 6 \text { hrs and clear liquids until } 4 \text { hrs prior to surgery } \\
\mathrm{CHO} \text { beverage and instructions provided (consume } 4 \text { hrs prior to surgery) }\end{array}$ \\
\hline \multirow{4}{*}{$\begin{array}{l}\text { Day of op (holding } \\
\text { area) }\end{array}$} & Preemptive analgesia \\
\hline & Acetaminophen $(1000 \mathrm{mg})$ and gabapentin $(300 \mathrm{mg})$ given orally in holding area \\
\hline & PONV risk assessment \\
\hline & Scopolamine patch placed if patient was at high risk of PONV and there was no contraindication \\
\hline \multirow[t]{4}{*}{ IOP } & Standardized anesthetic and analgesic protocol \\
\hline & $\begin{array}{l}\left.\text { TIVA with propofol ( } 50-100 \mu \mathrm{g} \cdot \mathrm{kg} \cdot \mathrm{min}^{-1}\right) \text {, ketamine }\left(0.1-0.5 \mathrm{mg} \cdot \mathrm{min}^{-1}\right) \text {, up to } 0.5 \mathrm{MAC} \text { INH as needed, but avoid } \mathrm{N}_{2} \mathrm{O} \\
\text { Dual anti-emetic prophylaxis therapy (dexamethasone } 4 \mathrm{or} 8 \mathrm{mg} \text { and ondansetron } 4 \mathrm{mg}) \\
\text { MMA: lidocaine bolus ( } 1 \mathrm{mg} \cdot \mathrm{kg}^{-1} \text { on induction) and infusion }\left(2 \mathrm{mg} \cdot \mathrm{kg}^{-1} \text { until closure of surgical incision); ketorolac ( } 15 \mathrm{or}\right. \\
30 \mathrm{mg} \text { during closure); acetaminophen ( } 1000 \mathrm{mg} \text { intravenously), if not given preop } \\
\text { Goal: normothermia }\left(36.0-37.0^{\circ} \mathrm{C} \text { ) and normovolemia (mild fluid restriction, approximately } 10-15 \mathrm{ml} \cdot \mathrm{kg}^{-1} \text { total) }\right. \\
\text { Antibiotic prophylaxis within } 60 \text { mins of incision }\end{array}$ \\
\hline & Standardized surgical protocol \\
\hline & $\begin{array}{l}\text { MIS approach } \\
\text { No surgical drain; no Foley catheter } \\
\text { Topical methylprednisolone prior to surgical closure }\end{array}$ \\
\hline \multirow[t]{6}{*}{ Postop } & Early oral intake \\
\hline & As soon as swallow evaluation complete and patient recovers from anesthesia \\
\hline & Early mobilization \\
\hline & Within 2 hrs of PACU arrival \\
\hline & Continue MMA \\
\hline & $\begin{array}{l}\text { Acetaminophen (1000 mg every } 6 \mathrm{hrs} \text { ); NSAIDs (meloxicam } 7.5 \mathrm{mg} \text { every } 12 \mathrm{hrs} \text { ); tramadol (50 mg oral, } \times 2 \text { doses as } \\
\text { needed); oxycodone ( } 5 \mathrm{mg} \text { ) for poorly controlled pain }\end{array}$ \\
\hline
\end{tabular}

$\mathrm{CHO}=$ carbohydrate; $\mathrm{MAC} \mathrm{INH}=$ minimum alveolar concentration, inhaled anesthetic; NPO = nil per os; TIVA = total intravenous anesthesia.

* Twenty-one standard, evidence-based elements are included.

years] $)(\mathrm{t}(31)=2.7 ; \mathrm{p}<0.05)$. The percentage of patients in each surgical cohort differed by sex, with more men undergoing $\mathrm{ACDF}(52 \%)$ compared to $\mathrm{CDA}\left(25 \% ; \chi^{2}=9.5\right.$ $[1, \mathrm{~N}=33], \mathrm{p}=0.002)$. The mean \pm SD duration of surgery was $73 \pm 21.9$ minutes for ACDF and $60 \pm 6.9$ minutes for CDA. There were no significant differences in duration of surgery $(\mathrm{t}(31)=1.64 ; \mathrm{p}=0.11)$ or estimated blood loss $(\mathrm{t}(31)=-0.43 ; \mathrm{p}=0.67)$ between the 2 surgical subtypes.

\section{Pathway Compliance}

We tracked 21 care elements. Overall pathway compliance was $85.6 \%$ (Fig. 1A). The median number of ERAS process elements provided per patient was 18 . There was no significant correlation between LOS and number of ERAS elements provided (Pearson's $r=-0.20$ ) (Fig. 1B). Items with the lowest compliance $(<70 \%)$ were use of topical methylprednisolone (used in $52.9 \%$ of cases), preoperative carbohydrate loading (61\%), use of ketorolac as part of the MMA regimen (62\%), and goal normothermia (65.5\%). Components with the highest compliance included use of acetaminophen (100\% of cases), multimodal anesthesia (100\% of cases), and MIS (no cases required conversion to open surgery).

\section{Primary and Secondary Outcomes}

The median LOS was 416 minutes (IQR 210-1643 minutes)-i.e., 6 hours, 56 minutes (IQR 3 hours, 30 minutes-27 hours, 23 minutes) (Fig. 2A). Eight patients (24\% of the cohort) required an extended stay longer than 23 hours. The median LOS for these patients was 1585 minutes (IQR 1423-1713 minutes)-i.e., 26 hours, 25 minutes (IQR 23 hours, 43 minutes-28 hours, 33 minutes) (Fig. 2B). Reasons for extended admission included pain $(n=4)$, dyspnea not requiring intervention $(\mathrm{n}=1)$, hypoxia requiring supplemental oxygen $(\mathrm{n}=1)$, hypertension $(\mathrm{n}=1)$, and dysphagia $(n=1)$. There were no readmissions within 90 days of discharge.

Overall, $12 \%$ of the cohort ( $\mathrm{n}=4$ patients) was opioid tolerant at the time of surgery, as defined by Centers for Disease Control criteria. ${ }^{6}$ Opioid-tolerant and opioid-naïve patients did not differ significantly on total intraoperative opioid consumption $(\mathrm{t}(31)=0.54 ; \mathrm{p}>0.05)$ or total PACU 

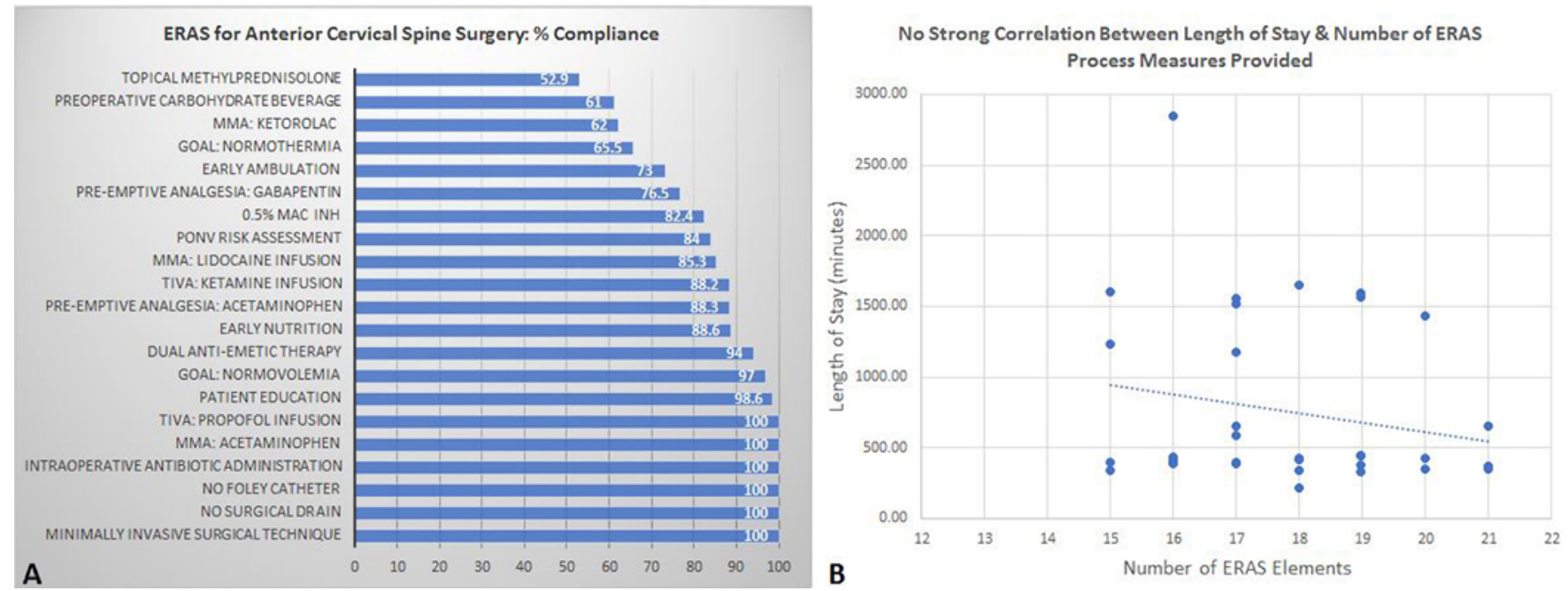

FIG. 1. A: ERAS for anterior cervical spine pathway compliance. Overall pathway compliance with included process measures was $85.6 \%$. MAC INH = minimum alveolar concentration, inhaled anesthetic; TIVA = total intravenous anesthesia. B: Chart showing that there was no strong association between LOS and number of ERAS process elements provided (Pearson's $r=-0.20$ ).

opioid consumption $(t(31)=0.95 ; p>0.05)$ (Fig. 3A). We were unable to formally analyze differences between opioid status and median LOS due to small sample sizes (Fig. 3B). Two of the patients admitted for an extended stay due to pain were opioid tolerant at baseline.

\section{Discussion}

ERAS pathways focus on optimizing patient recovery by reducing the global stress response to surgery. ${ }^{13}$ According to ERAS principles, the stress response is most effectively modulated by delivering evidence-based, procedure-specific interventions and minimizing variations in care. As the demand for spine surgery grows,${ }^{23}$ and practice patterns shift from inpatient to ambulatory settings, ${ }^{5}$ it becomes increasingly important to derive care models that facilitate safe, rapid discharge after spine surgery.

Our group previously reported our experience with an MIS-ERAS pathway for lumbar decompression. ${ }^{31}$ Using the lumbar decompression pathway as a template, we reviewed the evidence for components of care that positively affect outcomes after anterior cervical spine surgery, tailored the pathway, and then applied it to 33 patients undergoing elective ACDF or CDA with an MIS approach. Here we report a retrospective analysis of all cases performed in the first 6 months postimplementation, and found high
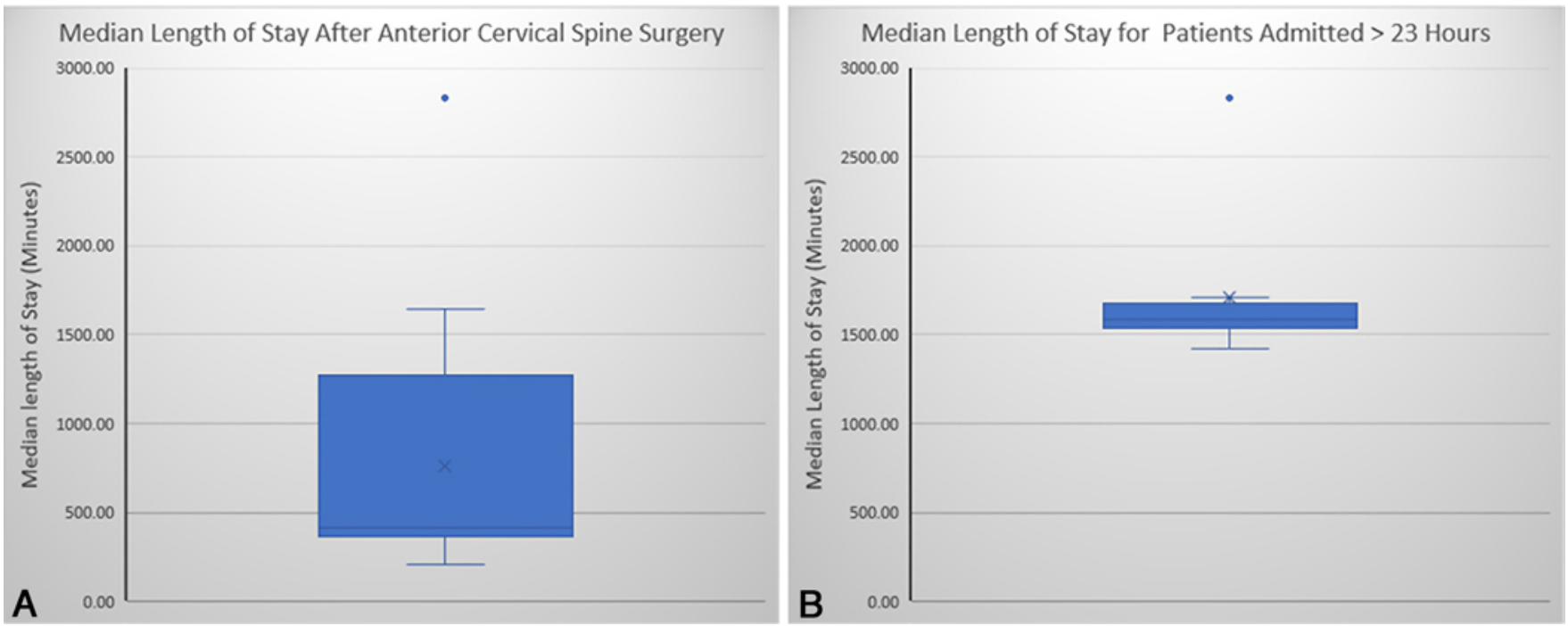

FIG. 2. A: The median LOS for the cohort was 416 minutes (IQR 210-1643 minutes)-i.e., 6 hours, 56 minutes (IQR 3 hours, 30 minutes-27 hours, 23 minutes). The mean \pm SD LOS for $75 \%$ of the cohort was $759 \pm 235$ minutes-i.e., 12 hours, 39 minutes \pm 3 hours, 55 minutes. B: The median LOS for 8 patients who required extended monitoring prior to discharge was 1585 minutes (IQR 1423-1713 minutes)-i.e., 26 hours, 25 minutes (IQR 23 hours, 43 minutes-28 hours, 33 minutes). 


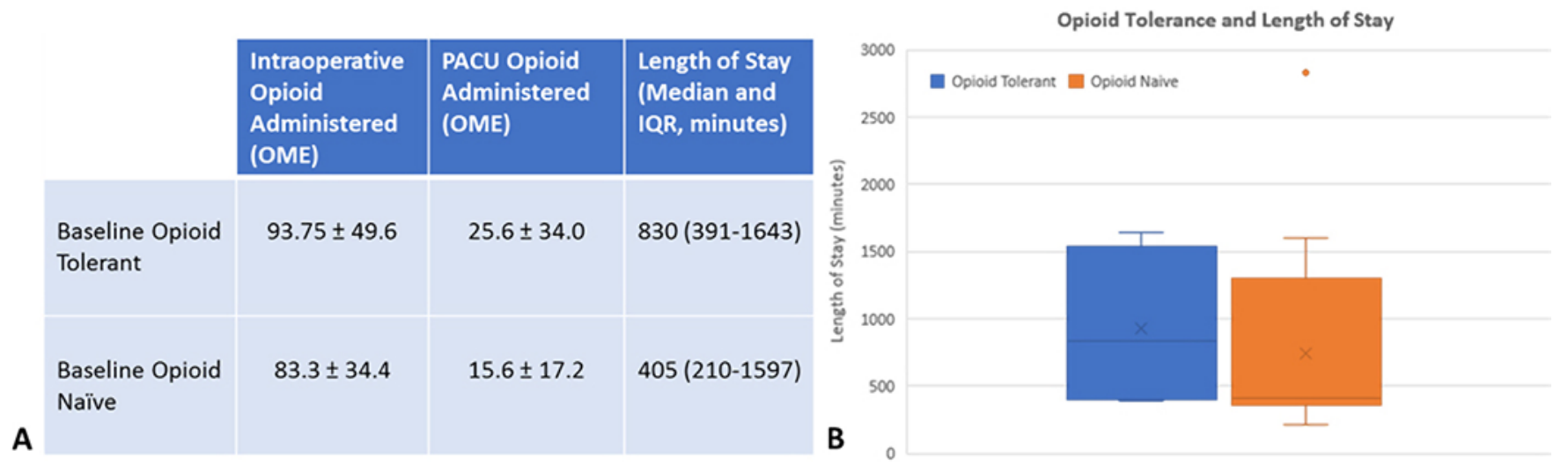

FIG. 3. A: Four patients ( $12 \%$ of the cohort) were opioid tolerant at the time of surgery. There were no significant differences between total intraoperative or PACU-administered opioid, or LOS between opioid-tolerant and opioid-naïve patients. B: LOS for patients who were opioid tolerant and opioid naïve at the time of surgery. OME = oral morphine equivalents.

pathway compliance, short LOS, minimal complications, and no readmissions within 90 days of surgery.

To date, the evidence to support ERAS for spine surgery has been poorly described. To our knowledge, there are only 3 studies in evidence in the published literature that report data on outcomes related to ERAS for spine surgery. The earliest featured an MIS approach for lumbar fusion, sedation anesthesia, and liposomal bupivacaine analgesia. ${ }^{35}$ The authors found improved functional scores and low rates of complications among 42 patients cared for on the pathway. The second study, by our group, included the full evidence base informing a comprehensive ERAS pathway for lumbar decompression. ${ }^{31} \mathrm{~A}$ retrospective analysis of 61 patients associated the pathway with short LOS, minimal complications, and no readmissions within 90 days of surgery. A third study compared outcomes after surgery for metastatic spine tumors before and after ERAS implementation. ${ }^{8}$ Although the pathway was associated with a trend toward better pain scores and decreased opioid consumption, there were no significant differences in other important outcomes (LOS, 30-day readmission, and 30-day complication rates).

We propose that the heterogeneity of spine surgery limits the appropriateness of a single ERAS pathway for all patients and procedures. Classic ERAS components, including opioid-sparing MMA, PONV prophylaxis, early mobilization, and omission of routine surgical drains and urinary catheters, are all well-established components of ERAS protocols. ${ }^{19}$ Many also show efficacy within the spine surgery literature and should be included in all ERAS for spine pathways. ${ }^{34}$ However, unique factors for recovery suggest that tailoring of pathways for individual spine surgery subtypes is more appropriate than a onesize-fits-all approach. This is highlighted by our current report-specifically, airway compromise is a rare but potentially life-threatening complication of anterior cervical spine surgery. Evidence that most complications occur in the immediate period after ACDF ( $<6$ hours) informed the decision to include a minimum PACU stay of 5 hours in our protocol. ${ }^{18}$ Other interventions that minimize this risk include restriction of intraoperative intravenous fluid administration $^{11}$ and topical methylprednisolone. ${ }^{26,37} \mathrm{We}$ included these 3 elements of care in our anterior cervical ERAS pathway, but not in our lumbar decompression pathway, based on procedure-specific benefits to recovery. Interestingly, the use of topical methylprednisolone was among the ERAS process elements with the lowest rates of compliance in our study. This result was surprising, in light of the importance of preventing airway compromise after anterior spine surgery.

Although the early respiratory complication rate is low, ${ }^{18}$ this rate is not zero, and it must be emphasized that early discharge after ACDF may not always be safe or feasible. Our final decision to discharge is made on a case-bycase basis, after consultation between the anesthesiologist and surgeon. We consider the complexity of surgery, as well as patient and social factors including difficulty of intubation and proximity of the discharge destination to a facility with an emergency room. All patients included in this study were discharged to locales within 20 minutes of an emergency room, and patients were counseled to present immediately in the event of any respiratory distress or compromise.

Other protocol elements, including MIS and NSAIDs, are less well supported or are more controversial. As a technique that minimizes tissue disruption, MIS may be particularly beneficial for anterior cervical procedures for minimizing postoperative pain burden and opioid requirements as well as reducing the surgical stress response. These concepts have been demonstrated in ERAS for colorectal surgery: in trials comparing open with laparoscopic techniques for colorectal resection, superior outcomes and lower serum markers of surgical stress are associated with less invasive (i.e., laparoscopic) surgical approaches. ${ }^{25,36}$ Additional procedure-specific data associate MIS using zeroprofile cages with less post-ACDF dysphagia ${ }^{28}$ and shorter operative duration. ${ }^{24}$ Prospective trials comparing MIS to open techniques are limited for cervical spine surgery at this time and are required before concluding the superiority of one technique within ERAS pathways.

The inclusion of NSAIDs in our pathway was based on an evaluation of risks and benefits of analgesia versus bleeding and delayed fusion. Recent systematic reviews suggest that NSAIDs have dose- and duration-dependent effects 
on fusion, and that a short course of a low-dose NSAID after spine fusion is reasonable. ${ }^{20,27}$ Important analgesic and opioid-sparing benefits of NSAIDs have been consistently reported after multiple surgical subtypes, including spine surgery. ${ }^{22,38}$ Interestingly, opioids have recently been implicated in delayed lumbar spine fusion in a rabbit model. ${ }^{12}$ Thus, avoiding NSAID analgesia in favor of opioids may not confer a protective benefit against delayed fusion. We included ketorolac, a mixed cyclooxygenase inhibitor, and did not find adverse effects related to bleeding, hematoma, or rates of fusion, albeit in a small sample size. However, more recent data suggest that selective cyclooxygenase- 2 inhibitors may ultimately be preferred, given minor effects on platelet function and perioperative bleeding. ${ }^{33}$

We found high overall compliance with the ERAS pathway. Individual process elements with the lowest compliance included NSAID provision as part of the MMA regimen, consumption of the preoperative carbohydrate-rich beverage, targeted intraoperative normothermia, and topical methylprednisolone. Because this was a retrospective study, we are unable to determine the reasons for poor compliance with these individual care elements. Possible explanations include patient contraindication or refusal, intervention not ordered (or not ordered in time to be provided), inconsistent documentation, ongoing concern regarding side effects of medications, and other overriding clinical decision-making. Improving overall compliance with an ERAS pathway is important for achieving better outcomes. As adherence to the number of process elements increases over time, the incidence of postoperative complications and readmission decreases. ${ }^{10}$ These data further associate a threshold of $>$ $70 \%$ pathway compliance with ERAS success, whereas adherence rates $<50 \%$ are associated with worse outcomes. We attribute our high compliance to several factors, including use of a small ERAS team whose members developed the pathway and were highly motivated to ensure success. Strategies to improve compliance with ERAS elements have been published, and include 1) use of a specialized working group to develop and implement the protocols; 2) continuous education for all practitioners; and 3) continuous audit and regular dissemination of results as part of an active ERAS research program..$^{15}$

Finally, we found that $12 \%$ of the cohort was opioid tolerant at the time of surgery. Over the past 10 years, multiple reports have concluded that there is a consistent association between preoperative opioid use and worse outcomes after spine surgery. These include higher risk of long-term opioid use/dependence, ${ }^{4}$ and higher pain and disability scores. ${ }^{17}$ Other data found that work status, higher preoperative disability scores, and pending litigation-but not preoperative opioid use-predicted poor outcomes. ${ }^{2}$ These results are difficult to reconcile, but may be explained (at least in part) by the choice of agent and duration of opioid treatment as well as other perioperative factors. These relationships were explored in a recent comparison of patients taking strong versus weak preoperative opioids, which found no association between opioid use and worse outcomes after ACDF.14

\section{Study Limitations}

There are several limitations to our present study. Be- cause this was a small, retrospective study, the strength of our analyses and conclusions is limited. Our study lacks a comparative cohort, so it is unknown whether the ERAS pathway, as implemented, is superior to institutional standard of care. This is a common problem in ERAS research and represents an important area for future study. We were unable to track reasons for failure to provide individual process elements, which may have resulted in an underestimation of elements of protocol compliance. Prospective data collection with the use of ERAS checklists may facilitate more accurate tracking of the indications for providing or withholding elements in the future. Our study was performed in an experienced center serviced by a small, well-organized team committed to ERAS processes and goals. This may limit the generalizability of our results to less specialized centers-but it also highlights the importance of an organized team approach for successful implementation of ERAS programs. Our facility and patients are based in a large urban setting. Given the importance of postoperative respiratory compromise and potential requirement for access to emergency care, early discharge may not be universally applicable or safe for all practice settings. Finally, all of our patients were classed as American Society of Anesthesiologists (ASA) grade I or II, so the pathway may not be universally applicable in a practice setting with higher patient comorbid burden.

\section{Conclusions}

We have developed an ERAS pathway for ACDF and CDA with the goal of facilitating safe, prompt discharge. Our ERAS pathway was associated with overall high compliance, minimal complications, and no readmissions within 90 days of surgery. Overall LOS was certainly increased by our requirement for a 5 -hour minimum observation in PACU, but we were able to identify 3 patients with potential respiratory compromise who were then admitted for further observation. This safety element of our pathway is unique to anterior cervical spine surgery and highlights the requirement to tailor ERAS pathways to spine surgery subtypes. Pain and respiratory compromise were both linked with extended LOS in this cohort. Further prospective studies are needed to confirm the potential benefits of ERAS for anterior cervical spine surgery, including longer-term complications, cost, and functional outcomes.

\section{References}

1. Ali ZS, Ma TS, Ozturk AK, Malhotra NR, Schuster JM, Marcotte PJ, et al: Pre-optimization of spinal surgery patients: development of a neurosurgical enhanced recovery after surgery (ERAS) protocol. Clin Neurol Neurosurg 164:142-153, 2018

2. Anderson PA, Subach BR, Riew KD: Predictors of outcome after anterior cervical discectomy and fusion: a multivariate analysis. Spine (Phila Pa 1976) 34:161-166, 2009

3. Apfel CC, Zhang K, George E, Shi S, Jalota L, Hornuss C, et al: Transdermal scopolamine for the prevention of postoperative nausea and vomiting: a systematic review and metaanalysis. Clin Ther 32:1987-2002, 2010

4. Armaghani SJ, Lee DS, Bible JE, Archer KR, Shau DN, Kay $\mathrm{H}$, et al: Preoperative opioid use and its association with perioperative opioid demand and postoperative opioid indepen- 
dence in patients undergoing spine surgery. Spine (Phila Pa 1976) 39:E1524-E1530, 2014

5. Baird EO, Egorova NN, McAnany SJ, Qureshi SA, Hecht AC, Cho SK: National trends in outpatient surgical treatment of degenerative cervical spine disease. Global Spine J 4:143-150, 2014

6. Dowell D, Haegerich TM, Chou R: CDC guideline for prescribing opioids for chronic pain-United States, 2016. MMWR Recomm Rep 65:1-49, 2016

7. Gan TJ, Diemunsch P, Habib AS, Kovac A, Kranke P, Meyer TA, et al: Consensus guidelines for the management of postoperative nausea and vomiting. Anesth Analg 118:85-113, 2014

8. Grasu RM, Cata JP, Dang AQ, Tatsui CE, Rhines LD, Hagan $\mathrm{KB}$, et al: Implementation of an Enhanced Recovery After Spine Surgery program at a large cancer center: a preliminary analysis. J Neurosurg Spine 29:588-598, 2018

9. Grosh T, Elkassabany NM: Enhanced recovery after shoulder arthroplasty. Anesthesiol Clin 36:417-430, 2018

10. Gustafsson UO, Hausel J, Thorell A, Ljungqvist O, Soop M, Nygren J: Adherence to the enhanced recovery after surgery protocol and outcomes after colorectal cancer surgery. Arch Surg 146:571-577, 2011

11. Hart RA, Dupaix JP, Rusa R, Kane MS, Volpi JD: Reduction of airway complications with fluid management protocol in patients undergoing cervical decompression and fusion across the cervicothoracic junction. Spine (Phila Pa 1976) 38:E1135-E1140, 2013

12. Jain N, Himed K, Toth JM, Briley KC, Phillips FM, Khan SN: Opioids delay healing of spinal fusion: a rabbit posterolateral lumbar fusion model. Spine J 18:1659-1668, 2018

13. Kehlet H: Multimodal approach to control postoperative pathophysiology and rehabilitation. Br J Anaesth 78:606617, 1997

14. Kelly MP, Anderson PA, Sasso RC, Riew KD: Preoperative opioid strength may not affect outcomes of anterior cervical procedures: a post hoc analysis of 2 prospective, randomized trials. J Neurosurg Spine 23:484-489, 2015

15. Khan S, Gatt M, Horgan A, Anderson I, MacFie J: Issues in Professional Practice: Guidelines for Implementation of Enhanced Recovery Protocols. London: Association of Surgeons of Great Britain and Ireland, 2009

16. Lamperti M, Tufegdzic B, Avitsian R: Management of complex spine surgery. Curr Opin Anaesthesiol 30:551-556, 2017

17. Lawrence JTR, London N, Bohlman HH, Chin KR: Preoperative narcotic use as a predictor of clinical outcome: results following anterior cervical arthrodesis. Spine (Phila Pa 1976) 33:2074-2078, 2008

18. Lied B, Sundseth J, Helseth E: Immediate (0-6 h), early (6-72 h) and late ( $>72 \mathrm{~h}$ ) complications after anterior cervical discectomy with fusion for cervical disc degeneration; discharge six hours after operation is feasible. Acta Neurochir (Wien) 150:111-118, 2008

19. Ljungqvist O, Scott M, Fearon KC: Enhanced recovery after surgery: a review. JAMA Surg 152:292-298, 2017

20. Marquez-Lara A, Hutchinson ID, Nuñez F Jr, Smith TL, Miller AN: Nonsteroidal anti-inflammatory drugs and bonehealing: a systematic review of research quality. JBJS Rev 4:e4, 2016

21. Marshall SI, Chung F: Discharge criteria and complications after ambulatory surgery. Anesth Analg 88:508-517, 1999

22. Martinez V, Beloeil H, Marret E, Fletcher D, Ravaud P, Trinquart L: Non-opioid analgesics in adults after major surgery: systematic review with network meta-analysis of randomized trials. Br J Anaesth 118:22-31, 2017

23. Oglesby M, Fineberg SJ, Patel AA, Pelton MA, Singh K: Epidemiological trends in cervical spine surgery for degenera- tive diseases between 2002 and 2009. Spine (Phila Pa 1976) 38:1226-1232, 2013

24. Saville P, Vaishnav AS, McAnany S, Gang CH, Qureshi SA: Predictive factors of post-operative dysphagia in single-level anterior cervical discectomy and fusion (ACDF). Spine (Phila Pa 1976) [epub ahead of print], 2018

25. Schwenk W, Haase O, Neudecker J, Müller JM: Short term benefits for laparoscopic colorectal resection. Cochrane Database Syst Rev (3):CD003145, 2005

26. Siasios I, Fountas K, Dimopoulos V, Pollina J: The role of steroid administration in the management of dysphagia in anterior cervical procedures. Neurosurg Rev 41:47-53, 2018

27. Sivaganesan A, Chotai S, White-Dzuro G, McGirt MJ, Devin CJ: The effect of NSAIDs on spinal fusion: a cross-disciplinary review of biochemical, animal, and human studies. Eur Spine J 26:2719-2728, 2017

28. Skovrlj B, Qureshi SA: Minimally invasive cervical spine surgery. J Neurosurg Sci 61:325-334, 2017

29. Soffin EM, Gibbons MM, Ko CY, Kates SL, Wick E, Cannesson M, et al: Evidence review conducted for the Agency for Healthcare Research and Quality Safety Program for Improving Surgical Care and Recovery: focus on anesthesiology for total knee arthroplasty. Anesth Analg 128:441-453, 2019

30. Soffin EM, Gibbons MM, Ko CY, Kates SL, Wick EC, Cannesson M, et al: Evidence review conducted for the Agency for Healthcare Research and Quality Safety Program for Improving Surgical Care and Recovery: focus on anesthesiology for total hip arthroplasty. Anesth Analg 128:454-465, 2019

31. Soffin EM, Vaishnav AS, Wetmore D, Barber L, Hill P, Gang $\mathrm{CH}$, et al: Design and implementation of an enhanced recovery after surgery (ERAS) program for minimally invasive lumbar decompression spine surgery: initial experience. Spine (Phila Pa 1976) [epub ahead of print], 2018

32. Soffin EM, YaDeau JT: Enhanced recovery after surgery for primary hip and knee arthroplasty: a review of the evidence. Br J Anaesth 117 (suppl 3):iii62-iii72, 2016

33. Teerawattananon C, Tantayakom P, Suwanawiboon B, Katchamart W: Risk of perioperative bleeding related to highly selective cyclooxygenase-2 inhibitors: a systematic review and meta-analysis. Semin Arthritis Rheum 46:520-528, 2017

34. Wainwright TW, Immins T, Middleton RG: Enhanced Recovery After Surgery (ERAS) and its applicability for major spine surgery. Best Pract Res Clin Anaesthesiol 30:91-102, 2016

35. Wang MY, Chang PY, Grossman J: Development of an Enhanced Recovery After Surgery (ERAS) approach for lumbar spinal fusion. J Neurosurg Spine 26:411-418, 2017

36. Watt DG, McSorley ST, Horgan PG, McMillan DC: Enhanced recovery after surgery: which components, if any, impact on the systemic inflammatory response following colorectal surgery? A systematic review. Medicine (Baltimore) 94:e1286, 2015

37. Zadegan SA, Jazayeri SB, Abedi A, Bonaki HN, Vaccaro AR, Rahimi-Movaghar V: Corticosteroid administration to prevent complications of anterior cervical spine fusion: a systematic review. Global Spine J 8:286-302, 2018

38. Zhang Z, Xu H, Zhang Y, Li W, Yang Y, Han T, et al: Nonsteroidal anti-inflammatory drugs for postoperative pain control after lumbar spine surgery: a meta-analysis of randomized controlled trials. J Clin Anesth 43:84-89, 2017

\section{Disclosures}

Dr. Albert has the following disclosures. He receives royalties from and/or does consulting for Zimmer Biomet and DePuy Synthes. He is a consultant for NuVasive. He receives royalties from JP Medical Publishers, Saunders/Mosby-Elsevier, and Thieme. He is on the Medical Advisory Board and/or does 
consulting for Facet Link, Gentis, and United Health Care. He has direct stock ownership in the following companies: Gentis, Vital 5, Bonovo Orthopedics Inc., Biomerix, InVivo Therapeutics, Spinicity, Crosstrees Medical, Paradigm Spine LLC, Invuity, ASIP, PMIG, and Pioneer. He is an employee of the Hospital for Special Surgery and of the Weill Cornell Medical College. He has received support for a non-study-related clinical or research effort that he oversees from PCORI, ISSG, and the Alan L. and Jacqueline B. Stuart Spine Research Center. He is a patent holder-US Patent 7,955,358 B2 (awarded June 7, 2011) and Patent US9717541 B2 (awarded August 1, 2017). He is also the past president of the Scoliosis Research Society. Dr. Qureshi has the following disclosures. He is a consultant for Zimmer Biomet, Stryker Spine, and Globus Medical, Inc. He receives royalties from Zimmer Biomet, Stryker Spine, and RTI. He has direct stock ownership in Vital 5 and in Avaz Surgical. He is a member of the board for Health Grades and for Minimally Invasive Spine Surgery Group.

\section{Author Contributions}

Conception and design: Soffin, Wetmore, Beckman, Albert, Qureshi. Acquisition of data: Soffin, Wetmore, Barber, Vaishnav, Qureshi. Analysis and interpretation of data: Soffin, Wetmore, Vaishnav, Qureshi. Drafting the article: Soffin, Wetmore, Barber, Beckman, Qureshi. Critically revising the article: Soffin, Wetmore, Barber, Vaishnav, Beckman, Albert, Qureshi. Reviewed submitted version of manuscript: all authors. Statistical analysis: Soffin. Administrative/technical/material support: Soffin, Barber, Vaishnav, Beckman, Albert, Gang, Qureshi. Study supervision: Soffin, Wetmore, Gang, Qureshi.

\section{Correspondence}

Ellen M. Soffin: Hospital for Special Surgery, New York, NY. soffine@hss.edu. 